Die Spektraltypen der 21 Sterne liegen im Bereich $\mathrm{O} 5$ bis $\mathrm{B} 1$ und sind von Petrie mit Hilfe von Linienintensitäten selbst klassifiziert worden.

Als mittlere Temperatur für den genannten Spektralbereich kann man $32000^{\circ} \mathrm{K}$ annehmen. Dieser Wert beruht auf den Temperaturbestimmungen, die Petrie aus dem Intensitätsverhältnis von Linien des neutralen und ionisierten Heliums vorgenommen hat. Er ist in ausreichender Übereinstimmung mit anderweitigen Ergebnissen. Im übrigen kommt es wegen der geringen Temperaturabhängigkeit von (1) für unseren Zweck auf eine genaue Kenntnis von $T$ überhaupt nicht an. Wir können daher auch unbedenklich mit einem Mittelwert für den gesamten Bereich rechnen, und es wird

$$
\begin{aligned}
& \log \frac{\mathrm{H}}{\mathrm{He}}=\frac{5}{2} \log \frac{A_{4340}}{A_{4471}}-0,51, \\
& \log \frac{\mathrm{H}}{\mathrm{He}}=\frac{5}{2} \log \frac{A_{4102}}{A_{4026}}-0,46 .
\end{aligned}
$$

Unglücklicherweise ist in den Spektren der OSterne die He I-Linie bei $\lambda=4026,36$ A ein Blend mit He II $\lambda=4025,60 \AA$, so daß sich aus $A 4102 / A 4026$ nur ein unterer Grenzwert für das Häufigkeitsverhältnis $\mathrm{H} / \mathrm{He}$ ergeben kann. Das Ausgangsmaterial und die ermittelten relativen Häufigkeiten enthält Tab. 1.

Die Schwankungen von log $\mathrm{H} / \mathrm{He}$ sind von Stern zu Stern teilweise erheblich. Besonders auffällig sind HD 47129, HD 188001 und HD 192639, bei denen Helium sogar häufiger oder mindestens genau so häufig wie Wasserstoff zu sein scheint. Wenn man auch den Einzelwerten der Tab. 1 kein allzu großes Gewicht beilegen darf - die Fehler in den Äquivalentbreiten gehen voll ein, der Faktor 2,5 wirkt aggravierend -, so ist ein solches Ergebnis nicht ohne weiteres völlig von der Hand zu weisen. Allerdings muß nach anderweitigen Erfahrungen für die große Stärke der Heliumlinien auch mit einem Úberanregungsphänomen in der Atmosphäre gerechnet werden. Im übrigen zeigen die $\log \mathrm{H} / \mathrm{He}$ keine Abhängigkeit von der spektralen Unterklasse oder der absoluten Helligkeit $M$, die in Tab. 1 ebenfalls gegeben sind und von $\mathrm{P}$ et $\mathrm{r}$ ie aus der Intensität der interstellaren Ca II-Linie $K$ bestimmt wurden. Die Mittelwerte aus den beiden Linienpaaren $\mathrm{H} \gamma / \mathrm{He} \mathrm{I} \lambda=4471 \AA$ und $\mathrm{H} \delta / \mathrm{He} \mathrm{I}$ $\dot{\lambda}=4026 \AA$ betragen 0,59 und 0,48. Der Unterschied entspricht wegen der erwähnten Störung von $\lambda=4026 \AA$ der Erwartung.

Der Mittelwert, der hier gefunden worden ist, bestätigt aufs neue die in der letzten Zeit mehrfach gemachte Feststellung, daß das Häufigkeitsverhältnis von Wasserstoff und Helium entlang der Hauptreihe von den $\mathrm{O}$ - bis mindestens $\mathrm{zu}$ den $\mathrm{G} 0$-Sternen einheitlich ist, an Hand vergrößerten Beobachtungsmaterials für die O-Sterne. Eine Reihe von Einzelbestimmungen des Verhältnisses $\mathrm{H} / \mathrm{He}$ ergab immer Werte von sehr ähnlicher Größe. Das hier mitgeteilte Ergebnis gilt für das obere Ende der Hauptreihe, für das untere Ende ihres bisher der Untersuchung zu- gänglich gewordenen Teils, eine eruptive Protuberanz der Sonne (G0), fand Uns öl d ${ }^{4} \log \mathrm{H} / \mathrm{He}=0,74 \pm 0,5$.

Auf die kosmogonischen Konsequenzen dieser Gleichförmigkeit ihrer chemischen Zusammensetzung ist kürzlich vor allem von ten $\mathrm{Bruggencat} \mathrm{e}^{5}$ und $\mathrm{Uns}$ öl $\mathrm{d}^{6}$ hingewiesen worden.

4 A. Unsöld, Z. Astrophysik 24, 22 [1947].

5 P. ten B r ug g encat e, Z. Astrophysik 24, 48 [1947].

${ }^{6}$ A. U n söld, Z. Astrophysik 24, 306 [1948].

\section{Die Mesonenausbeute beim Beschuß von leichten Kernen mit $\alpha$-Teilchen}

Von Heinz Koppe

Max-Planck-Institut für Physik, Göttingen

(Z. Naturforschg. 3 a, 251--252 [1948]; eingeg. am 21. Juni 1948)

Mittels des neuen Berkeley-Betatrons ist es möglich gewesen, durch Beschuß von leichten Kernen (insbesondere C) mit $\boldsymbol{\alpha}$-Teilchen von etwa $380 \mathrm{MeV}$ Mesonen $\mathrm{zu}$ erzeugen. Im folgenden soll eine einfache Methode angegeben werden, nach der sich die dabei zu erwartende Ausbeute abschätzen läßt.

Beim Stoß eines Kernes mit der Massenzahl $M_{1}$ und der kinetischen Energie $E$ auf einen ruhenden Kern mit der Masse $M_{2}$ entsteht zunächst ein Zwischenkern mit der Masse $M=M_{1}+M_{2}$, dem die Anregungsenergie pro Nucleon

$$
U=\frac{M_{2}}{M^{2}} E
$$

zur Verfügung steht. Nach einer bekannten Beziehung ${ }^{\mathbf{1}}$ hat der $\mathrm{Zwischenkern} \mathrm{dann} \mathrm{die} \mathrm{Temperatur}$

$$
T_{0}=3,8 \sqrt{U} \text {. }
$$

Dabei wird unter $T$ das Produkt aus $k$ und der absoluten Temperatur verstanden. Gl. (2) liefert $T$ in $\mathrm{MeV}$, wenn man $U$ in $\mathrm{MeV}$ einsetzt.

Bei den Berkeley-Experimenten ergeben sich so Temperaturen von der Größenordnung $10 \mathrm{MeV}$. Für das Weitere ist wichtig, daß man bei derartig hohen Temperaturen nicht mehr mit konstanten Teilchenzahlen rechnen darf, sondern die Möglichkeit der Paarerzeugung berücksichtigen muß. Man erhält dann für jede Teilchensorte rein statistisch eine bestimmte Gleichgewichtskonzentration. Diese Erscheinung wurde von Houtermans und Jensen ${ }^{2}$,Dissoziation des Vakuums" und von Kop p e ${ }^{3}$,Paarentartung“" genannt und bisher vor allem im Hinblick auf die Er. zeugung von Elektron-Positron-Paaren untersucht. Dieselben Überlegungen lassen sich natürlich auch auf die Erzeugung von Mesonenpaaren anwenden. Die thermische Erzeugung von Teilchen setzt ein, sobald die Temperatur von der Größenordnung der Ruheenergie der Teilchen wird. Da die Ruheenergie der

1 Vgl. E. B a g g e, Ann. Physik (5) 33, 389 [1938]; Fußnote auf S. 399 .

2 F. Houtermans u. H. Jensen, Z. Naturforschg. 2 a, 146 [1947].

${ }^{3}$ H. K o p p e, Ann. Physik (6) 2, 103 [1948]. 
Mesonen etwa $150 \mathrm{MeV}$ beträgf, werden wir nur mit einer sehr geringen Mesonenbildung zu rechnen haben und müssen in diesem Fall die von Koppe ${ }^{3}$ abgeleiteten Gleichungen für ,tiefe“ Temperaturen benutzen. Für die Elektronen mit einer Ruheenergie von $0,5 \mathrm{MeV}$ befinden wir uns dagegen längst im Gebiet vollständiger Paarentartung, in dem sich das Elektronengas praktisch wie die Hohlraumstrahlung benimmt. Die Energiedichte ist z. B. gegeben durch

$$
u=2 \cdot \frac{7}{8} \frac{\pi^{2}}{15 c^{3} \hbar^{3}} T^{4} .
$$

Dabei kommt der Faktor 2 daher, daß man es jetzt mit zwei Teilchen (Elektronen und Positronen) zu tun hat, während sich der Faktor $7 / 8$ aus dem Unterschied zwischen Bose-Einstein- und Fermi-Dirac-Statistik ergibt. Der Rest ist die bekannte Energiedichte der Hohlraumstrahlung. Da ferner die Geschwindigkeit der Elektronen von der Größenordnung $c$ sein wird, ist auch der durch die Elektronen bedingte Energiefluß gegenüber der Lichtquantenstrahlung um diesen Faktor erhöht, so daß man beide in eins zusammenfassen und einfach nach dem Stefan-Boltzmannschen Gesetz unter Hinzufügung eines Faktors $(1+7 / 4)=11 / 4$ berechnen kann. Der Strahlungsverlust des Zwischenkernes mit der Oberfläche $O$ ist dann gegeben durch

$$
q=\frac{11 \pi^{2}}{240} \frac{1}{c^{2} \hbar^{3}} T^{4} O
$$

Für den Temperaturverlauf ergibt sich unter Berücksichtigung von (2) die Beziehung

$$
\frac{\partial}{\partial t} M \frac{T^{2}}{3,8^{2}}=-\frac{11 \pi^{2}}{240} \frac{O T^{4}}{c^{2} \hbar^{3}} .
$$

Integration liefert

$$
\frac{1}{T}=\sqrt{\frac{1}{T_{0}{ }^{2}}+2 B t} ; \quad B=\frac{3,8^{2} \cdot 11 \pi^{2}}{480 \cdot c^{2} \hbar^{3}} \frac{O}{M} .
$$

Natürlich wird sich der Zwischenkern nicht nur infolge des Strahlungsverlustes abkühlen, sondern auch durch Expansion, d. h. indem er auseinanderfliegt. Aber da sich die schweren Nucleonen verhältnismäßig langsam bewegen, wird er inzwischen schon durch Ausstrahlung soweit abgekühlt sein, daß ohnehin keine Mesonen mehr emittiert werden können.

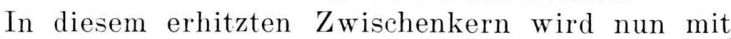
einer bestimmten Wahrscheinlichkeit ein Meson vorhanden sein. Diese ist als Funktion der Temperatur und der kinetischen Energie des Mesons gegeben $\operatorname{durch}^{4}$

$$
n(\varepsilon)=\frac{(2 \mu)^{3 / 2}}{\pi^{2} \hbar^{3}} \varepsilon^{1 / 2}\left\{e^{\left(\mu c^{2}+\varepsilon\right) / T}+1\right\}^{-1}
$$

( $\mu=$ Mesonenmasse). Daraus ergibt sich die Anzahl der in der Zeiteinheit herausfliegenden Mesonen zu

$$
v=\frac{O \mu T^{2}}{\pi^{2} \hbar^{3}} e^{-\mu c^{2} / T} .
$$

Die Ausbeute an Mesonen ist dann gegeben durch

$$
\eta=\int_{0}^{\infty} v(T) d t=\frac{O \mu}{\pi^{2} \hbar^{3}} \int_{0}^{\infty} T^{2} e^{-\mu c^{2} \sqrt{1 / T_{0}^{2}+2 B t}} d t .
$$

Unter dem Integral kann man $T^{2}$ als langsam veränderlich durch $T_{0}{ }^{2}$ ersetzen und außerdem die Wurzel nach $t$ entwickeln. Es ergibt sich

$$
\eta=0,031 T_{0} M e^{-\mu c^{2} / T_{0}} .
$$

Mit den oben angegebenen Werten liefert das Stoßausbeuten $\eta=1,7 \cdot 10^{-4}$.

Die gröbsten Vereinfachungen sind bei der vorliegenden Rechnung bei der Abschätzung von $T_{0}$ gemacht worden, indem angenommen wurde, daß die Energie des aufprallenden $\alpha$-Teilchens sich zunächst einmal restlos und gleichmäßig auf den ganzen $\mathrm{Z}_{w}$ ischenkern verteilt. Aus der Theorie der Kernverdampfung ${ }^{5}$ ist bekannt, daß zunächst einmal ein kleiner Teil des Zwischenkernes entsprechend höher erhitzt wird. Das würde zu einer Vergrößerung von $T_{\mathbf{0}}$ und damit von $\eta$ führen. Anderseits kann $T_{0}$ dadurch kleiner werden, daß bereits vor Erreichen der Höchsttemperatur Energie abgestrahlt wird.

$4 \mathrm{Vgl}$. Anm. 3, Gl. (6) und (13').

5 W. H e i s e $n$ b e r g, Ber. Verh. sächs. Akad. Wiss.

\begin{tabular}{|c|c|c|c|}
\hline Substanz & $\begin{array}{l}\text { Insgesamt zur Ver- } \\
\text { fügung stehende } \\
\text { Menge in mCurie }\end{array}$ & $\begin{array}{l}\text { Material- } \\
\text { kosten pro } \\
\text { mCurie } \\
\text { ca. DM }\end{array}$ & $\begin{array}{l}\text { Transport- } \\
\text { kosten } \\
\text { ca. DM }\end{array}$ \\
\hline $\mathrm{p}^{32}$ & monatlich $\quad 2-\tilde{5}$ & 40.- & 5.50 \\
\hline$S^{35}$ & $\begin{array}{r}\text { vierteljährlich } 1 \mathrm{mC} \\
\text { in } 20 \mathrm{~g} \mathrm{KCl}\end{array}$ & 40.- & 5.50 \\
\hline$I^{131}$ & halbmonatlich 1 & $40 .-$ & $130 .-$ \\
\hline $\bar{K}^{42}$ & wöchentlich & $40 .-$ & $130 .-$ \\
\hline $\mathrm{Cu}^{6 \mathrm{t}}$ & wöchentlich & 40. & $130 .-$ \\
\hline $\mathrm{Na}^{24}$ & wöchentlich & $40 .-$ & $130 .-$ \\
\hline
\end{tabular}
Leipzig, math.-physische Kl. 89, 369 [1937].

\section{NACHRICHTEN}

\section{Radioaktive Isotope}

Dank den Bemühungen des British Research-Branch können radioaktive Isotope für wissenschaftliche Arbeiten über die JEIA bezogen werden.

Lieferbar sind vorerst nur in beschränkten Mengen:

Anfragen und Bestellungen sind an die $\mathrm{B}$ i o chemische Abteilung der Medizin ischen Forschungsanstalt der Max-Planck-Gesellschaft, (20b), Göttingen, Bunsenstr. 10, zu richten. 